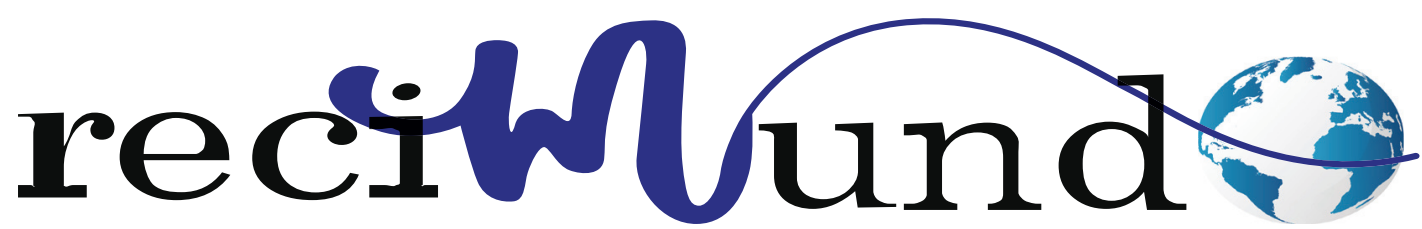

Revista Científica Mundo de la Investigación y el Conocimiento

DOI: 10.26820/recimundo/4.(4).noviembre.2020.364-373

URL: http://recimundo.com/index.php/es/article/view/966

EDITORIAL: Saberes del Conocimiento

REVISTA: RECIMUNDO

ISSN: 2588-073X

TIPO DE INVESTIGACIÓN: Artículo de revisión

Código UNESCO: Ciencias Médicas

PAGINAS: $364-373$

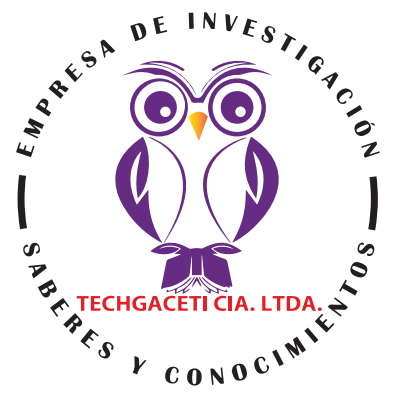

\title{
Cirugía mínimamente invasiva de la hernia inguinal en tiempos de COVID-19
}

Minimally invasive surgery of inguinal hernia in times of COVID-19 Cirurgia minimamente invasiva de hérnia inguinal em tempos de COVID-19 Danilo Fernando Tiban Jaque; Paúl Darío Hidalgo Mina²; Negus Felipe Ojeda Correa³

RECIBIDO: 10/07/2020 ACEPTADO: 02/09/2020 PUBLICADO: 10/12/2020

1. Médico General; Investigador Independiente; Guayaquil, Ecuador; danilitotibancito@gmail.com; iD https://orcid.org/00000002-5867-4644

2. Médico General; Hospital Sur Delfina Torres de Concha Esmeraldas; Ecuador; daryloates1983@gmail.com; (D) https:// orcid.org/0000-0003-4612-2495

3. Diplomado de Bioseguridad ante Covid-19 Universidad Politécnica de Colombia; Médico; Curso de Inglés en Kaplan In-ternational English Toronto- Canadá; Médico Residente de Cuidados Intensivos e Intermedios Hospital Sur Delfina Torres de Concha; Investigador Independiente; Esmeraldas, Ecuador; negus6000@hotmail.com; (iD https://orcid.org/0000-00033904-0010

\section{CORRESPONDENCIA \\ Danilo Fernando Tiban Jaque \\ danilitotibancito@gmail.com \\ Guayaquil, Ecuador}

() RECIMUNDO; Editorial Saberes del Conocimiento, 2020 


\section{RESUMEN}

Dentro del contexto pandémico COVID-19, las cirugías para reparar las hernias inguinales (HI) no han sido prioritarias y muchas veces descartadas de la programación quirúrgica, salvo aquellos casos específicos de urgencia que ameriten la pronta intervención. En algunos centros quirúrgicos, la técnica laparoscópica, que a pesar de ser mínimamente invasiva y que permite al paciente permanecer poco tiempo en las instalaciones sanitarias disminuyendo el riesgo de contagio por Sar-Cov-2, es considerada más riesgosa que otras técnicas quirúrgicas porque genera muchas partículas de aerosol al realizarse el neumoperitoneo, y por esta razón se prescinden de su uso. Sin embargo, las recomendaciones de expertos sobre el uso de sistema de filtración y otras condiciones de bioseguridad, ha permitido su uso con éxito en el Reino Unido y Europa, inclusive la han considerado más segura que otras técnicas de cirugía en estos tiempos de COVID-19.

Palabras clave: Hernia, laparoscopia, cirugía, COVID-19, Sar-Cov-2

\section{ABSTRACT}

Within the COVID-19 pandemic context, surgeries to repair inguinal hernias $(\mathrm{IH})$ have not been a priority and are often ruled out from the surgical programming, except for those specific emergency cases that warrant prompt intervention. In some surgical centers, the laparoscopic technique, which despite being minimally invasive and which allows the patient to stay for a short time in health facilities, reducing the risk of infection by Sar-Cov-2, is considered more risky than other surgical techniques because it generates many aerosol particles when pneumoperitoneum is performed, and for this reason they are not used. However, expert recommendations on the use of a filtration system and other biosafety conditions have allowed its successful use in the United Kingdom and Europe, and have even considered it safer than other surgical techniques in these times of COVID- 19.

Keywords: Hernia, laparoscopy, surgery, COVID-19, Sar-Cov-2.

\section{RESUMO}

No contexto da pandemia COVID-19, as cirurgias para reparar hérnias inguinais $(\mathrm{IH})$ não têm sido uma prioridade e são frequentemente excluídas da programação cirúrgica, excepto para os casos de emergência específicos que justificam uma intervenção rápida. Em alguns centros cirúrgicos, a técnica laparoscópica, que apesar de ser minimamente invasiva e que permite ao paciente permanecer por um curto período de tempo nas instalações de saúde, reduzindo o risco de infecção por Sar-Cov-2, é considerada mais arriscada do que outras técnicas cirúrgicas porque gera muitas partículas de aerossol quando o pneumoperitôneo é realizado, e por esta razão não são utilizadas. No entanto, as recomendações de peritos sobre a utilização de um sistema de filtração e outras condições de biossegurança permitiram a sua utilização bem sucedida no Reino Unido e na Europa, e consideraram-no mesmo mais seguro do que outras técnicas cirúrgicas nestes tempos da COVID- 19.

Palavras-chave: Hérnia, laparoscopia, cirurgia, COVID-19, Sar-Cov-2. 


\section{Introducción}

La aceptación y adaptación de los cambios que se han dado en todos los centros de salud y hospitalarios del mundo a raíz de la pandemia COVID-19, no ha sido fácil de sobrellevar para el personal que laboran en ellos. En esta creciente emergencia, y en muchos casos con recursos limitados, se han reestructurado en un tiempo record la organización en todos los niveles de atención en un gran esfuerzo por preservar la salud y vida de los pacientes.

En cirugía esta rápida restructuración ha generado incertidumbres con respecto a la práctica, a la seguridad del personal que allí labora, y sobre la atención general del paciente (Pryor, 2020). Mucha de esta incertidumbre procede de la falta de evidencia científica en momentos caóticos que requieren de respuestas rápidas a la emergencia sin que esta suponga un riesgo de exposición que se convierta en trasmisión del Sar-Cov-2 durante las intervenciones quirúrgicas. Pero también, la incertidumbre se deriva en la reorganización de los protocolos y manejos de los cribados que se han dado sobre la marcha para la toma de decisiones sobre priorización de cirugías, y sobre la práctica misma en algunas técnicas quirúrgicas, como es el caso de la laparotomía, porque esta ha sido foco de polémicas en la comunidad médico-científica por ser uno de los procedimientos que generan más partículas de aerosol al realizarse el neumoperitoneo (López et al, 2020).

En cuanto a la priorización de las cirugías, las intervenciones quirúrgicas parar reparar hernias, tiene una larga lista de espera en muchos lugares del mundo a causa de ésta pandemia, aunque ya de por sí las cirugías para reparación de hernia no complejas, y mientras que el paciente no sea un bebe o niño, no son prioritarias frente a una inmensa mayoría de cirugías de mayor urgencia (de la Cuadra et al, 2018).
Es un hecho que en medio de la pandemia COVID-19, el procedimiento quirúrgico para reparar una hernia inguinal $(\mathrm{HI})$, es mucho menos prioritario si además el paciente no presenta ningún síntoma y si ésta es pequeña, y por ello tiende a ser pospuesta (American College of Surgeons, 2020).

Si aproximadamente, el 5\% de la población mundial padece de $\mathrm{HI}$, en esta actual creciente morbilidad a causa de las medidas de priorización obligada por la necesidad de atender la emergencia pandémica, no es de extrañarse como efecto futuro a mediano plazo, un aumento en la tasa de morbimortalidad por las complicaciones urgentes derivadas del aplazamiento prioritario (American College of Surgeons, 2020).

El presente artículo, describe la situación actual de las cirugías para reparar HI, y más específicamente, la técnica laparoscópica como cirugía para tratar esta patología en medio de la pandemia por Sar-Cov-2. Para ello, se presenta información clave sobre la patología, como su definición, epidemiología y los tipos de tratamientos quirúrgicos aplicados, haciendo énfasis en la intervención quirúrgica laparoscópica como intervención mínimamente invasiva para tratarla, para finalmente llegar al contexto de la intervención quirúrgica de la $\mathrm{HI}$ en tiempos de COVID-19.

\section{Metodología}

A través de una investigación documental descriptiva se llevó a cabo una búsqueda de información más actualizada a través de diferentes bases de datos digitales como PubMed, ScienceDirect, Google Scholar, y en sitios web de la Cochrane Library, la OMS, la European Association for Endoscopic Surgery (EAES) y la Society of American Gastrointestinal and Endoscopic Surgeons (SAGES), a través de las cuales se recogen las recomendaciones y evidencia científica de expertos médicos, que estuvieran al día con la situación pandémica por Sar-Cov-2 
en el ambiro quirúrgico, específicamente, para cirugías de la pared abdominal como tratamiento para $\mathrm{HI}$.

Una vez seleccionada la documentación, se procedió al análisis comparativo, reflexivo y evaluativo, para la posterior organización y estructuración de la información que se manifiesta en el presente artículo. En el mismo se desmenuza la información para conocer desde las partes hasta el todo del tema a tratar. De esta manera, se exponen conceptos de relevancia científica que permiten vías de soluciones y alternativas en el manejo de este tipo de intervenciones quirúrgicas mínimamente invasivas en medio de esta actual pandemia.

\section{Resultados}

\section{Hernia Inguinal}

La hernia como patología se presenta cuando algún órgano o tejido protruye fuera de la cavidad donde está. Generalmente, las de mayor incidencia son las de la cavidad abdominal, donde algunos órganos se salen de esta a través de algunos orificios que existen naturalmente en su pared, sin comprometer la integridad del peritoneo y de la piel, sino que más bien se distienden en forma de saco (de la Cuadra et al, 2018). Este debilitamiento de la fascia que permite que se produzca la hernia, puede ser de origen congénito o adquirido (obesidad, edad avanzada, esfuerzo excesivo, trauma,).

De este tipo de hernias, la más común es la Inguinal la cual se da con una incidencia del $75 \%$ del $5 \%$ de casos que se producen en la población mundial. Los hombre tienen 25 veces más riesgo de padecer este tipo que las mujeres, y en los niños es 5 veces más frecuente (Cochrane, 2018).

La manifestación clínica la HI es un abultamiento en la ingle (puede ser en el escroto), o el área abdominal, y comúnmente aumenta en tamaño cuando se tose o se hace un esfuerzo. Puede ser dolorosa o no y/o puede presentar coloración en la piel. El dolor abdominal agudo y vómito pueden indicar que el intestino se ha deslizado a través del saco de la hernia y está estrangulado, convirtiéndose en una emergencia médica requiriendo tratamiento quirúrgico inmediato (Brunicardi et al, 2005).

Si no hubiese síntoma alguno, esperar en alerta es una alternativa con ayuda de fajas, vendas o cinturones hechos para aplicar presión sobre una hernia, pero que generalmente necesitan un ajuste correcto. No obstante, hay que tomar en cuenta que el estrangulamiento de la misma (según la evaluación clínica) es muy probable si se espera mucho tiempo, lo que pudiera devenir en un posible daño a los nervios testiculares y encarcelación, si fuera el caso, o a otras complicaciones como la obstrucción intestinal (de la Cuadra et al, 2018).

Es por ello que, aunque no parezca ser un problema grave al momento del diagnóstico, las HI no deben descuidarse, y el médico tratante debe también considerar otros factores en cada caso individual (edad, sexo, condiciones de salud del paciente). En el caso de las hernias femorales, estas deben ser reparadas siempre a la prontitud, debido "al alto riesgo (30-40\%) de encarcelamiento y de estrangulación del intestino en un periodo de dos años a partir de la fecha del diagnóstico" (Brunicardi et al, 2005).

Los tipos de HI pueden ser:

- Indirectas, cuando el contenido de ésta se sale por el anillo inguinal interno.

- Directa, cuando su contenido protruye por la pared posterior.

- Femorales, cuando su tamaño es del anillo femoral.

El único tratamiento para reparar una $\mathrm{HI}$ es por medio de la cirugía, y en la actualidad existen diversas y efectivas técnicas quirúr-

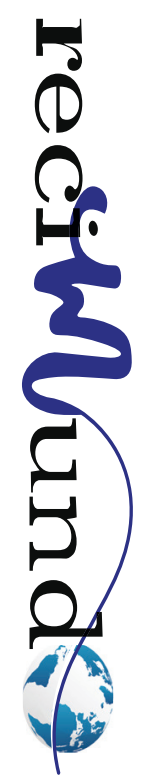


gicas alrededor del mundo, cuya superioridad de una sobre la otra depende del dominio del cirujano, de los materiales que estén al alcance, y la condición del paciente mismo. Finalmente, la elección de una u otra técnica, radica en los criterios de riesgos de recidiva, costo-beneficio, técnicas locales al alcance y los deseos del paciente (SoHaH, 2020).

Las técnicas desarrolladas para la reparación de HI son la Herniorrafia, la Hernioplastia Protésica y la Laparoscópica (con malla). La elección dependerá de los criterios descritos en el párrafo anterior, por lo que conocer el caso particular del paciente, la situación local del centro de salud que éste a su alcance, junto con la capacitación técnica del cirujano, será lo que finalmente defina la opción más adecuada al caso. Dado que la Herniorrafia, que ha sido la primera técnica comprobada por muchos años de práctica, podría servir para ciertos pacientes cuya musculatura y tamaño y localización de la hernia, esta pueda ser aplicada sin complicaciones ni recidiva (de la Cuadra et al, 2018).

No obstante, cada vez hay más evidencias de que las técnicas sin tensión, que utilizan las prótesis sintéticas, tienen menos recidivas (reducidas al 30\%-50\%) y un postoperatorio inmediato menos doloroso que la Herniorrafia, lo cual hace que el cirujano esté preparado para la vanguardia (ver figura 1), ya sea para Hernioplastia Protésica o la técnica Laparoscópica, porque esta evidencia está más relacionada con el uso de la malla que con el método de su colocación, ya que "la mayoría de los ensayos individuales fueron demasiado pequeños como para demostrar beneficios claros de un tipo de reparación quirúrgica sobre otro" (Cochrane, 2018).
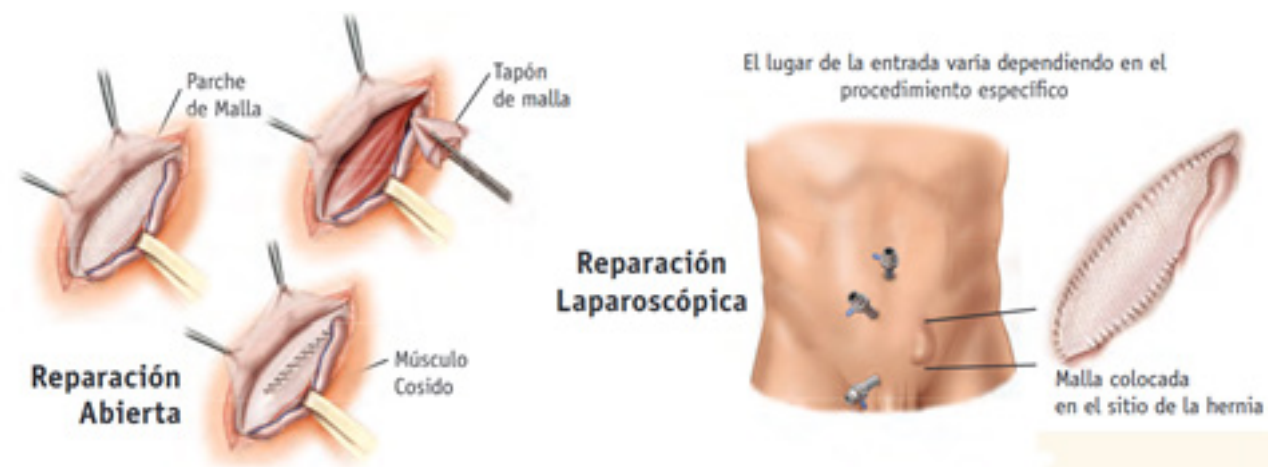

Figura 1. Técnicas quirúrgicas para reparación de Hernia Inguinal

Fuente: American College of Surgeons (2014) (ob. cit.)

\section{Técnica quirúrgica cerrada vs abierta en tiempos de COVID-19}

Hasta el momento presente, las evidencias han indicado que entre las rutas de trasmisión del SARS-Cov-2, la vía aérea, a través de las partículas virales en forma de aerosol, es la más aceptada (OMS, 2020). Por ello, cuando Zhen $\mathrm{MH}$ et al (2020) plantean la posibilidad de contagio de COVID-19 por medio de la exposición al humo quirúrgico que se produce durante una cirugía laparoscópica, basados en estudios sobre algunos sistemas que producen una nube de humo en donde se ha detectado VPH y VIH, .todas las sociedades científicas de cirugía se apegaron a la recomendación de no usar la técnica de laparoscopia, a menos que el beneficio clínico supere en mucho el riesgo de contagio por SARS-Cov-2. 
La cirugía laparoscópica frente a la cirugía con técnica abierta, ha tenido su ventaja por ser mínimamente invasiva y por lo cual los pacientes no permanecían en las instalaciones hospitalarias por mucho tiempo. Pero en estos tiempos de COVID-19, lejos de ser esto una ventaja obvia para que el paciente no estuviera expuesto mucho tiempo en las instalaciones sanitarias, se desvaneció ante el hecho fáctico de que es uno de los procedimientos que generan más partículas de aerosol, porque requerir realizar un neumoperitoneo. Desde entonces, se ha cuestionado su ventaja. Sumado a ello, está la energía usada (que también es usada en la técnica abierta) que aumenta la producción de humo quirúrgico, el aerosol de baja temperatura generado por el bisturí ultrasónico, la apertura de las válvulas de los trocares y la extracción de las piezas quirúrgicas, entre otros, también se suma al humo del quirófano que pone en riesgo al personal al posible aerosol con SARS-Cov-2 proveniente del neumoperitoneo (Mallick et al, 2020). En ese momento inicial de la pandemia, el punto en cuestión era saber cuánta capacidad de protección tenía el personal de quirófano contra las partículas producidas durante la cirugía laparoscópica, sabiendo que el electrocauterio produce partículas menores a $0.1 \mu \mathrm{m}$, el láser utilizado para la ablación tisular genera partículas mayores $0.3 \mu \mathrm{m}$, y el bisturí ultrasónico (armónico), cuya superficie entra en contacto directo con tejido, sangre y sus bioproductos, produce partículas entre $0.35 \mu \mathrm{m}$ y $6.5 \mu \mathrm{m}$, (ver figura 2), mientras se buscaba evidencia de presencia de SARS-Cov-2 en el líquido peritoneal, tejido graso y epiplón (Safari $\mathrm{S}$ et al, 2020).

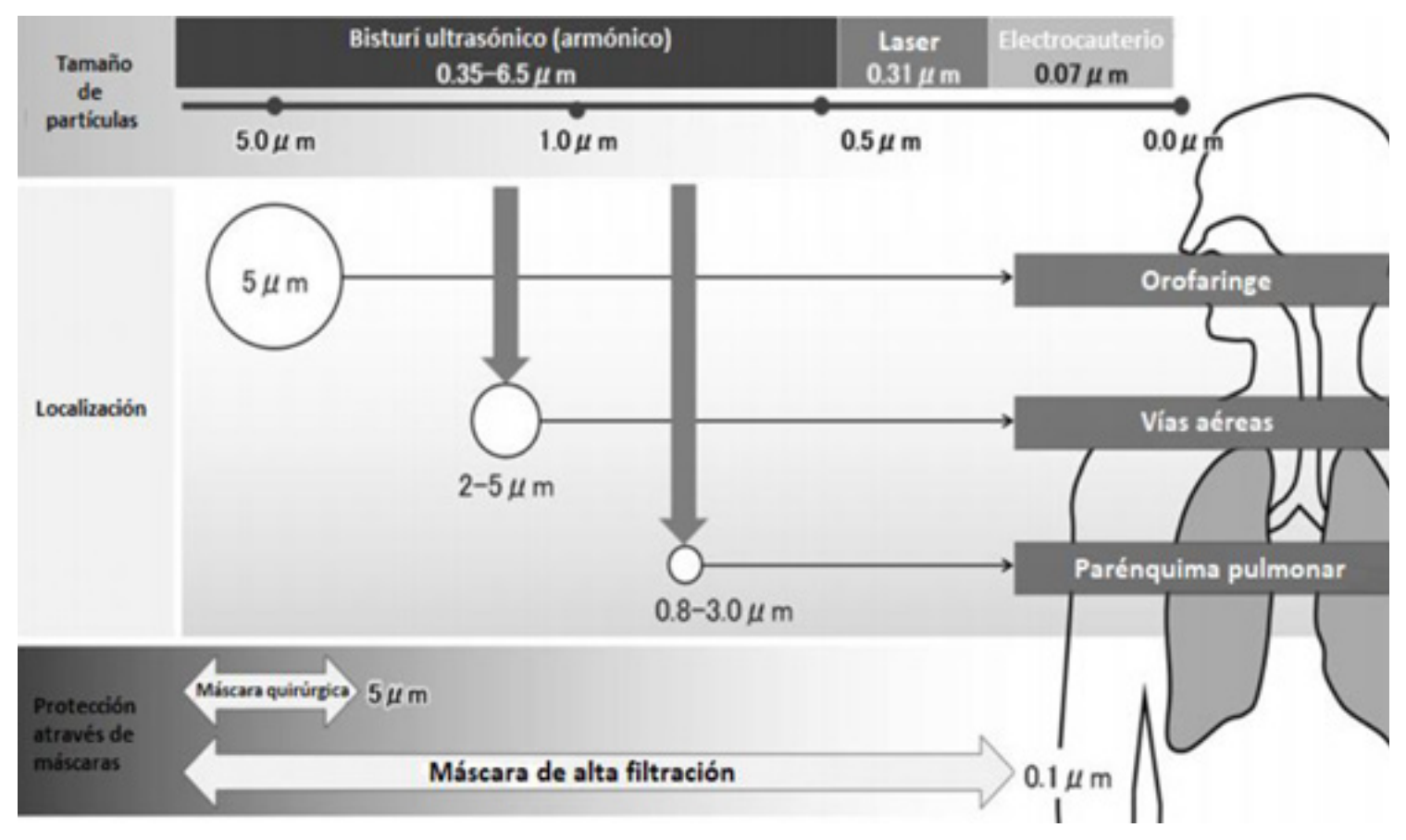

Figura 2. Capacidad de protección de los cirujanos de acuerdo al tamaño de partículas producidas por los dispositivos quirúrgicos

Fuente: IETSI (2020) (ob. cit). 
Sin embargo, al poco tiempo, la Sociedad Americana de Cirujanos Gastrointestinales y Endoscópicos (SAGES), recomendó el uso de la técnica laparoscópica bajo ciertas condiciones y recomendaciones:

1. El personal quirúrgico debe usar el correcto equipo de protección personal.

2. Minimizar el uso de dispositivos de energía avanzado durante la cirugía.

3. Aumentar el uso de dispositivos de succión para absorber el humo y aerosoles durante la operación.

4. Configurar a nivel mínimo posible las unidades de electrocirugía.

5. Evitar el uso de insufladores de neumoperitoneo de 2 vías, para así disminuir la colonización de patógenos del aerosol que puede circular en las adyacencias del neumoperitoneo o el insuflador.

6. Usar filtros de humo quirúrgico para partículas virales. Debe evacuarse todo el neumoperitoneo mediante este sistema de filtración antes del cierre, en la extracción del trócar, durante la extracción de la muestra o cuando se pase a la técnica abierta.

7. Se recomienda el uso de quirófano de presión negativa (si estuviera disponible).

Por supuesto, estas recomendaciones fueron seguidas por la mayoría de los hospitales y sociedades de cirujanos en todo el mundo, pero como no en todos los lugares se puede contar con dichas condiciones, el uso de cirugía mínimamente invasiva se han suspendido o pospuesto, más que por la incertidumbre que supone la falta de evidencia, ha sido por miedo al contagio (Mallick, Odejinmi, \& Clark, 2020).

Actualmente, no se cuenta con evidencia suficiente que confirme la trasmisión del virus a través del humo quirúrgico, ni siquiera los estudios que han demostrado la presencia de VPH, VIH, y VHB en este ambiente, con los que se quiera extrapolar la posibilidad de contagio del SARS-CoV-2, no han podido documentar la trasmisión de estos por el humo durante la cirugía, y sin embargo, se ha justificado preferir la técnica quirúrgica por vía abierta que la laparoscopia, a pesar de la falta de evidencia.(López et al, 2020).

No obstante, actualmente en Europa y el Reino Unido se continúa haciendo cirugía laparoscópica regularmente, sin reporte de contagio de SARS-CoV-2 por exposición al humo quirúrgico (Mallick, Odejinmi, \& Clark, 2020).

De hecho, la EAES ha llegado a considerarla como la técnica quirúrgica más segura en estos tiempos de COVID-19, gracias al sistema de filtrado del humo quirúrgico, y porque además el personal de cirugía no está expuesto a la cavidad abierta de forma directa. También se protege al paciente negativo en la PCR por SARS-CoV-2, ya que esta cirugía mínimamente invasiva, permite su pronta alta disminuyéndose su exposición en el ambiente hospitalario. Y aunque fuera un paciente COVID-19, también sería una ventaja su recuperación inmediata, pensando en la seguridad del personal asistencial (López et al, 2020).

Esto es un indicador claro de que sí se puede realizar cirugía laparoscópica en pacientes COVID-19, si se cumplen las condiciones y recomendaciones generales de las sociedades científicas del mundo, y por supuesto, si el caso quirúrgico amerita el riesgo y el recurso local de la institución dónde es atendido.

\section{La cirugía laparoscópica para reparar HI en tiempos de COVID-19}

Ante la pandemia por SARS-Cov-2, los hospitales y centros clínicos de todo el mundo enfrentan un desafío único para adaptarse a esta nueva realidad. Los protocolos y prácticas se han alterado para dar respuestas rápidas y de contención de la actual pandemia. La OMS como autoridad mun- 
dial de la salud, ha marcado lineamientos a los que se han apegado la mayoría de las asociaciones científico-médicas del mundo, como la European Association for Endoscopic Surgery (EAES), la Society of American Gastrointestinal and Endoscopic Surgeons (SAGES), y todas las demás asociaciones de cirujanos alrededor del mundo que apegados a dichos lineamientos, han proporcionado a sus localidades orientaciones sobre el manejo en el área quirúrgica adaptado a las necesidades y recursos disponibles.

La directiva constante que se mantiene en continuo llamamiento es: reducir los procedimientos quirúrgicos electivos. El cómo se va a manejar estos escenarios de priorización donde se contemplan las intervenciones urgentes y electivas no demorables, dependerá de una individualización de casos basados en un diagnóstico y criterio médico lo más certero posible, considerando la política de la institución de salud local (Pryor, 2020).

La cirugía para reparar la $\mathrm{HI}$ en un paciente descartado como COVID-19, que la misma no esté atascada, y que además el paciente haya elegido el procedimiento laparoscópico como opción quirúrgica, quedaría pospuesta en algunos lugares del mundo (Mallick et al, 2020).

Por no ser una emergencia por la cual arriesgar al paciente al potencial contagio en las instalaciones hospitalarias, y considerando el exceso de trabajo para el personal de salud, este tipo de intervención queda postergada. Sin embargo, si la HI estuviera en proceso de estrangulamiento, esto pasaría a ser una emergencia que debe ser resuelta, ya sea por la vía mínimamente invasiva o abierta (esto dependerá de las políticas y recursos de la institución hospitalaria), obedeciendo el protocolo de procedimientos quirúrgicos enmarcados dentro de la pandemia COVID-19 (SAGES, 2020).
Ciertamente, es muy difícil definir la urgencia médica en la programación de las cirugías electivas, porque si bien es cierto que se puede posponer de forma indefinida algunos casos, otros vienen asociados a una enfermedad que evoluciona como las enfermedades vasculares, o el cáncer o la insuficiencia orgánica (American College of Surgeons, 2020). Es por ello, que la decisión de cancelar, posponer o realizar un procedimiento quirúrgico debe tomarse considerando muchos factores que se derivan del caso mismo, ya que, como ya se ejemplifico en párrafos anteriores, retrasar algunos casos significa correr el riesgo de que reaparezcan como una emergencia grave, muy difícil de manejar.

A continuación, se hace un resumen de algunas recomendaciones dadas por la SAGES y EAES, que se están adoptando de manera general en la mayoría de los países:

- Todos los casos quirúrgicos y endoscópicos electivos deben posponerse en el actual momento, hasta que se observe el pico de la pandemia. Según la logística local, estas decisiones deben tomarse basándose en la carga de COVID-19, recursos, y sólo atender aquellos casos cuya necesidad sea inminentemente mortal.

- Los Centros quirúrgicos y hospitales deben satisfacer las necesidades médicas de los pacientes considerando los recursos y logísticas disponibles.

- Los procedimientos planteados deben ser determinados por un personal administrativo consciente de las limitaciones de la institución (cama, personal, suministro), y que se preocupe por el bienestar y seguridad de la población en general.

- Al calcular el riesgo para el paciente se debe añadir una evaluación del riesgo real de hacer la cirugía y el riesgo real en demorarla. Así mismo, se añade la expectativa de demora de 6 a 8 semanas con un posible entorno con menos

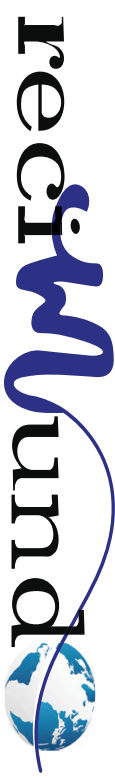


incidencia de COVID-19.

- - Se debe proceder con las recomendaciones de bioseguridad, en el contexto COVID-19, en el procedimiento quirúrgico tratando de disminuir los aerosoles en la medida de lo posible. Las cirugías laparoscópicas debe atender estrictamente a las recomendaciones dadas por los expertos.

Cabe destacar que entre los casos quirúrgicos considerados no esenciales en las anteriores recomendaciones, se encuentra la cirugía de la pared abdominal, principalmente, hernias. De hecho se hace el señalamiento que en una situación normal, fuera del contexto COVID-19, "la patología herniaria es una de las cirugías más frecuentes y tiene las listas de espera más largas" (SAGES, 2020).

Por otro lado, las hernias complejas de la pared abdominal, generalmente se presentan en pacientes con alta morbilidad asociada, con una calidad de vida deficiente, secundaria a su hernia. Por eso, "suelen requerir una prehabilitación, que en algunos casos puede ser ambulatoria (rehabilitación respiratoria, toxina botulínica, adelgazamiento), pero en otros casos puede requerir hospitalización (neumoperitoneo progresivo)" (SAGES, 2020).

La cirugía para estos pacientes, no puede posponerse indefinidamente y deben tener un espacio en la de procedimientos prioritarios, tanto en el quirófano como en la $\mathrm{UCl}$ tras la reanimación postoperatorio (SAGES, 2020).

A pesar de que en la mayoría de los centros quirúrgicos y hospitalario del mundo con un sistema de salud pública con cobertura universal y limitaciones, la cirugía mínimamente invasiva para reparar $\mathrm{HI}$ no es prioritaria, y además, por ser laparoscópica está contraindicada, quedando pospuesta en la mayoría de los casos, cabe decir que en establecimientos de salud privados, cuya capacidad para atender con recursos y personal suficiente y capacitado, la realizan con regularidad, atendiendo a las recomendaciones científicas e implantando el uso de sistema de evacuación de humos o filtración (Pryor, 2020).

\section{Conclusiones}

En medio de la pandemia COVID-19 tanto las cirugías mínimamente invasivas (laparoscópicas) como las abiertas para reparar HI, pasan a ser parte de los procedimientos postergados hasta observar la curva de incidencia, en la mayoría de los hospitales y centros quirúrgicos del mundo, por recomendaciones de la OMS y otras asociaciones de expertos cirujanos.

No obstante, no hay que perder en consideración que el aplazamiento hasta después de la pandemia de este tipo de patología, puede llegar a generar problemas importantes a nivel organizativo con los posibles crecientes casos de complicaciones y su creciente tasa de morbimortalidad.

Hablar de cirugía laparoscópica para HI en los actuales momentos parece inconcebible en algunas partes del mundo, porque por un lado, la técnica quirúrgica fue calificada como riesgosa por algunos estudios (Zhen $\mathrm{MH}$ et al, 2020), dada la cantidad de humo que ella supone y por los equipos quirúrgicos (laser, bisturí ultrasónico, electrocauterio) que se suman a este, y por el otro, la patología misma no es considerada prioritaria en la actual realidad de emergencia mundial por COVID-19 que acapara todo los recursos materiales y humanos.

Ante la falta de evidencia científica suficiente que corrobore la trasmisión del SARS-Cov-2 a través del humo quirúrgico, en muchos lugares del mundo como en Europa y el Reino Unido, se realizan cirugías laparoscópicas sin reportes de contagios hasta el momento, pero atendiendo a las observaciones y recomendaciones científicas sobre cómo 
eliminar humo del neumoperitoneo de forma segura mediante un sistema de filtración antes del cierre, la extracción del trocar, la extracción de la muestra o la conversión a la técnica abierta, entre otras medidas de bioseguridad, que involucran también al proceso de anestesia.

\section{Bibliografía}

American College of Surgeons. (Mayo de 2014). División of Education. Obtenido de Hernia: Inguinal/ Femoral: https://www.facs.org/ /media/files/education/patient\%20ed/herniaespanol.ashx

American College of Surgeons. (17 de Marzo de 2020). COVID-19: Guidance for Triage of Non-Emergent Surgical Procedures. Obtenido de https://www.facs.org/covid-19/clinical-guidance/ triage

Brunicardi et al. (2005). Principios de Cirugía (8th ed.). New York, NY: McGraw Hill.

Cochrane. (2018). Procedimientos de cirugía abierta para la hernia incisional. Obtenido de https://www. cochrane.org/es/CD006438/COLOCA_procedimientos-de-cirugia-abierta-para-la-hernia-incisional

de la Cuadra et al. (2018). El Libro de la Hernia Inguinal. Santiiago: de la Cuadra - Korn.

IETSI. (2020). Recomendaciones para Realizar Procedimientos Quirúrgicos en el Marco de la Pandemia por COVID-19. Lima: EsSalud.

López et al. (2020). Abdominal wall surgery after SARS-CoV-2: Time to reestablish postponed non-essential procedures? Elsevier, 98(9), 507509.
Mallick, R., Odejinmi, F., \& Clark, T. (2020). COVID-19 pandemic and gynaecological laparoscopic surgery: knowns and unknowns. Facts Views Vis Obgyn, 12(1), 7-11.

OMS. (2020). Manejo clínico de la COVID-19. Organización Mundial de la Salud 2020.

Pryor, A. (30 de Marzo de 2020). SAGES. Obtenido de SAGES and EAES Recommendations Regarding Surgical Response to COVID-19 Crisis: https://www.sages.org/recommendations-surgical-response-covid-19/

Safari S et al. (2020). Abdominal surgery in patients with COVID-19: detection of SARS-CoV-2 in abdominal and adipose tissues. Ann Surg, 272(2), 253256.

SAGES. (17 de Marzo de 2020). COVID-19: Guidance for Triage of Non-Emergent Surgical Procedures. Obtenido de Society of American Gastrointestinal and Endoscopic Surgeons (SAGES): https:// www.facs.org/covid-19/clinical-guidance/triage

Schmedt CG, Sauerland S, \& Bittner R. (2005). Comparación de procedimientos endoscópicos vs. Lichtenstein y otras técnicas de malla abierta para la reparación de la hernia inguinal. un meta-análisis de ensayos clínicos controlados aleatorios. Endoscopía Quirúrgica, 19, 188-199.

SoHaH. (Noviembre de 2020). Sociedad Hispanoamericana de Hernia. Obtenido de COVID-19 y Cirugía de la Pared Abdominal: https://www.sohah. org/covid-19-y-cirugia-de-la-pared-abdominal/

Zhen MH, Boni L, \& Fingerhut A. (2020). Minimally invasive surgery and the novel coronavirus outbreak: lessons learned in China and Italy. Ann Surg, 272(1).

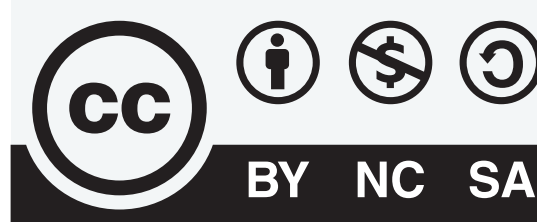

CREATIVE COMMONS RECONOCIMIENTO-NOCOMERCIAL-COMPARTIRIGUAL 4.0.

\section{CITAR ESTE ARTICULO:}

Tibán Jaque, D. F., Hidalgo Mina, P. D., \& Ojeda Correa, N. F. (2021). Cirugía mínimamente invasiva de la hernia inguinal en tiempos de COVID-19. RECIMUNDO, 4(4), 364-373. https://doi.org/10.26820/recimundo/4.(4).noviembre.2020.364-373 